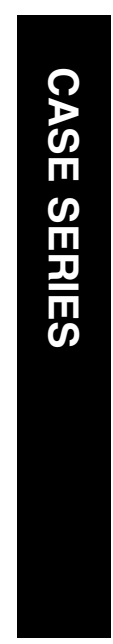

\section{Ocular perfusion pressure and glaucoma: the Beijing Eye Study}

$L X u^{1}, Y X$ Wang $^{1}$ and JB Jonas ${ }^{1,2}$

\section{Abstract}

Purpose IThe purpose of the study was to perfusion pressure and open-angle glaucoma
${ }^{1}$ Beijing Institute of Ophthalmology, Beijing Tongren Hospital, Capital Medical University, Beijing, China

${ }^{2}$ Department of Ophthalmology, Medical Faculty Mannheim of the Ruprecht-Karls-University of Heidelberg, Mannheim, Germany

Correspondence: $\mathrm{L} X \mathrm{X}$ and JB Jonas,

Beijing Institute of Ophthalmology,

17 Hougou Lane, Chong Nei Street, Beijing 100005, China Tel: + 49621383 2652;

Fax: +49621383 3803 .

E-mail: Jost.Jonas@

augen.ma.uni-heidelberg.de

Received: 18 June 2008

Accepted in revised form: 5

October 2008

Published online:

7 November 2008

Proprietary interest: None examine a relationship between ocular in a population-based setting in China.

Methods The Beijing Eye Study 2006 is a population-based investigation, which included 3251 subjects (response rate: $73.2 \%$ ). Mean age was $60.4 \pm 10.0$ years (range, 45-89 years). Glaucoma was defined by a glaucomatous appearance of the optic disc. Results Neither in univariate nor in multivariate statistical analysis, systolic blood pressure, diastolic blood pressure, mean blood pressure, and ocular perfusion pressure were significantly associated with the prevalence of open-angle glaucoma. It also holds true if the whole glaucoma group was differentiated into a normal-pressure glaucoma group and a high-pressure glaucoma group.

Conclusions In contrast to earlier population-based studies on western societies, the prevalence of open-glaucoma (also when differentiated into normal-pressure and high-pressure glaucomas) was not associated with the ocular perfusion pressure. The reasons for the discrepancy may be that most of the glaucoma patients in this study were untreated; that more than $80 \%$ of the glaucoma subjects of the present investigation had an intraocular pressure measurement $<22 \mathrm{mmHg}$; and differences in the definition of glaucoma. Eye (2009) 23, 734-736; doi:10.1038/eye.2008.342; published online 7 November 2008

Keywords: glaucoma; intraocular pressure; blood pressure; ocular perfusion pressure

\section{Introduction}

Earlier studies have suggested that a low ocular perfusion pressure defined as the difference of blood pressure minus intraocular pressure may be one of the risk factors for chronic open-angle glaucoma. ${ }^{1-4}$ It was the purpose of this study to reinvestigate this relationship between perfusion pressure and glaucoma in a population-based setting in China.

\section{Materials and methods}

The Beijing Eye Study is a population-based study in Northern China, described in detail recently. ${ }^{5}$ The Medical Ethics Committee of the Beijing Tongren Hospital approved the study protocol and all participants gave informed consent. Out of 5324 eligible individuals, 4439 individuals participated in the survey of 2001 (response rate: $83.4 \%$ ). In 2006, the study was repeated with 3251 subjects participating (response rate: $73.2 \%$ ). Mean age was $60.4 \pm 10.0$ years (range, 45-89 years). Besides a detailed ophthalmologic examination, the arterial blood pressure was measured by the Riva-Rocci method. Glaucoma was defined by a glaucomatous appearance of the optic disc as described recently. ${ }^{5}$ The height of intraocular pressure and presence of visual field defects were no criteria for the diagnosis of glaucoma.

\section{Results}

In the survey 2006, blood pressure measurements were available for $3222(99.1 \%)$ subjects, out of whom 76 (2.4\%) subjects showed a glaucomatous optic nerve damage and open anterior chamber angles. Out of these 76 patients with chronic open-angle glaucoma, 11 (15\%) subjects were treated by topical medical therapy only; 25 (33\%) additional patients had undergone laser therapy or glaucoma surgery. At the time of the survey, $63(83 \%)$ of the open-angle glaucoma subjects had an intraocular pressure measurement of $21 \mathrm{~mm} \mathrm{Hg}$ or less. In univariate analysis, the prevalence of 
glaucoma was significantly associated with intraocular pressure $(P<0.001$, odds ratio (OR):1.24; 95\% confidence interval (CI):1.16-1.32), age $(P<0.001$; OR: $1.07 ; 95 \% \mathrm{CI}$ : 1.04-1.09), gender (male ; $P=0.001$; OR: $0.47 ; 95 \% \mathrm{CI}$ : $0.29-0.75)$, and myopic refractive error $(P=0.006$; OR:0.91; 95\% CI: 0.84-0.97). It was not significantly associated with systolic blood pressure $(P=0.29$; OR: 1.01; 95\% CI: 0.99-1.03), diastolic blood pressure $(P=0.12$; OR: 0.97; 95\% CI:0.93-1.01), and mean blood pressure $(P=0.74$; OR: $0.99 ; 95 \% \mathrm{CI}$ : 0.96-1.03). Performing a binary regression analysis in a stepwise manner with the presence of chronic open-angle glaucoma as dependent variable and intraocular pressure, gender, and age as independent variables showed that the prevalence of open-angle glaucoma was significantly associated with intraocular pressure $(P<0.001)$, age $(P<0.001)$, and gender $(P=0.024)$. In a next step of the statistical analysis, the independent parameters, such as intraocular pressure, age, gender, and refractive error, were included in the analysis, and all four parameters were significantly associated with open-angle glaucoma (intraocular pressure $P<0.001$; age $P<0.001$; gender $P=0.015$; and refractive error $P=0.005)$. In a third step of the statistical analysis, in which the blood pressure measurements were added to the list of independent variables (although the blood pressure measurements were not significantly associated with the prevalence of open-angle glaucoma in univariate analysis), neither systolic blood pressure $(P=0.33)$ nor diastolic blood pressure $(P=0.11)$ nor the mean blood pressure $(P=0.70)$ was significantly associated with the prevalence of open-angle glaucoma. If additionally, the statistical analysis included the ocular perfusion pressure (defined as difference of systolic blood pressure minus intraocular pressure $(P=0.38)$; defined as difference of diastolic blood pressure minus intraocular pressure $(P=0.29)$; or defined as difference of mean blood pressure minus intraocular pressure $(P=0.28))$, none of these three variables was significantly associated with the prevalence of open-angle glaucoma. If the whole glaucoma population was divided into a subgroup with medical treatment and a subgroup without medical treatment, again the prevalence of openangle glaucoma was not associated with the blood pressure measurements nor with the perfusion pressure.

In an additional step, the whole open-angle glaucoma group was differentiated into subjects with normal intraocular pressure measurements ('normal-pressure glaucoma group') and subjects with an intraocular pressure $>21 \mathrm{mmHg}$ ('high-pressure glaucoma group'). In multivariate analysis including the normal-pressure glaucoma group, the presence of glaucoma was significantly associated with higher age $(P<0.001)$, higher intraocular pressure $(P=0.003)$, gender (male;
$P=0.033)$, and myopic refractive error $(P=0.002)$. It was not significantly associated with systolic blood pressure $(P=0.93)$, diastolic blood pressure $(P=0.58)$, mean blood pressure $(P=0.60)$, and the ocular perfusion pressure (difference of intraocular pressure to systolic blood pressure, $P=0.76$; to diastolic blood pressure, $P=0.53$; and to mean blood pressure, $P=0.60$ ). In a similar manner, none of the blood pressure related parameters was significantly $(P>0.10)$ associated with the presence of 'high-pressure glaucoma'. If both glaucoma groups were further subdivided into subjects treated vs subjects without treatment (normal-pressure glaucoma group, $n=19$ vs $n=41$ and high-pressure glaucoma group, $n=5$ vs $n=8$ ), the subgroup became too small for a meaningful statistical analysis.

\section{Conclusions}

The results suggest that in adult Chinese living the Greater Beijing area, the prevalence of chronic openangle glaucoma was not markedly associated with blood pressure values nor with the ocular perfusion pressure. It is in contrast with earlier investigations, such as the Rotterdam Study, ${ }^{2}$ the Barbados Eye Study, ${ }^{1}$ and others, ${ }^{3,6,7}$ which found a significant association between ocular perfusion pressure and open-angle glaucoma. The reasons for the discrepancy between the other studies mentioned and our study may be that about $47 \%$ of the open-glaucoma glaucoma subjects in this study were not on medical treatment, and that more than $80 \%$ of the glaucoma subjects of the present investigation had an intraocular pressure measurement of $21 \mathrm{mmHg}$ or less. Another reason for the discrepancies may be that the relatively small number of glaucoma patients in our study does not allow a detailed statistical analysis in subgroups. Yet another reason for the discrepancy in results could be that the criteria for glaucoma diagnosis were different in this as well as in earlier studies. Most of the earlier studies have relied on visual fields for diagnosis, whereas this study relied only on the optic nerve head appearance. Glaucoma in a patient with small discs may be difficult to diagnose without a visual field. Thus, there may be a systematic bias (based on different types of optic discs) in glaucoma detection in this study compared with earlier studies. Additional reasons for differences between the studies may refer to ethnic differences, differences in lifestyle, and genetic background.

\section{Acknowledgements}

This study was Supported by the National Key Laboratory Fund, Beijing, China. 


\section{References}

1 Leske MC, Wu SY, Nemesure B, Henis A. Incident openangle glaucoma and blood pressure. Arch Ophthalmol 2002, 120: 954-959.

2 Hulsman CA, Vingerling JR, Hofman A, Witteman JC, de Jong PT. Blood pressure, arterial stiffness, and open-angle glaucoma: the Rotterdam study. Arch Ophthalmol 2007; 125: 805-812.

3 Leske MC, Heijl A, Hyman L, Bengtsson B, Dong L, Yang Z, EMGT Group. Predictors of Long-term Progression in the Early Manifest Glaucoma Trial. Ophthalmology 2007; 114: 1965-1972.
4 Leske MC, Wu SY, Hennis A, Honkanen R, Nemesure B, BES Study Group. Risk factors for incident open-angle glaucoma: the Barbados Eye Studies. Ophthalmology 2008; 115: 85-93.

$5 \mathrm{Xu}$ L, Wang $\mathrm{Y}$, Wang S, Wang $\mathrm{Y}$, Jonas JB. High myopia and glaucoma susceptibility: the Beijing Eye Study. Ophthalmology 2007; 114: 216-220.

6 Bonomi L, Marchini G, Marraffa M, Bernardi P, Morbio R, Varotto A. Vascular risk factors for primary open angle glaucoma: the Egna-Neumarkt Study. Ophthalmology 2000; 107: 1287-1293.

7 Sommer A. Glaucoma risk factors observed in the Baltimore Eye Survey. Curr Opin Ophthalmol 1996; 7: 93-98. 